\title{
Root-Knot Nematodes in Golf Course Greens of the Western United States
}

Michael A. McClure, School of Plant Sciences, University of Arizona, Tucson 85721; Claudia Nischwitz, Department of Biology, Utah State University, Logan 84322; Andrea M. Skantar, United States Department of Agriculture-Agricultural Research Service Nematology Laboratory, Beltsville, MD 20705; Mark E. Schmitt, School of Plant Sciences, University of Arizona; and Sergei A. Subbotin, California Department of Food and Agriculture, Sacramento 95832

\begin{abstract}
McClure, M. A., Nischwitz, C., Skantar, A. M., Schmitt, M. E., and Subbotin, S. A. 2012. Root-knot nematodes in golf course greens of the western United States. Plant Dis. 96:635-647.

A survey of 238 golf courses in 10 states of the western United States found root-knot nematodes (Meloidogyne spp.) in 60\% of the putting greens sampled. Sequence and phylogenetic analyses of $18 \mathrm{~S}$ rRNA, D2-D3 of 28S rRNA, internal transcribed spacer-rRNA, and mitochondrial DNA gene sequences were used to identify specimens from 110 golf courses. The most common species, Meloidogyne naasi, was found in 58 golf courses distributed from Southern California to Washington in the coastal or cooler areas of those states. In the warmer regions of the Southwest, M. marylandi was recovered from 38 golf courses and M. graminis from 11 golf courses. This constitutes the first

Utah, and the first report of M. graminis in Arizona, Hawaii, and Nevada. Two golf courses in Washington were infested with M. minor, the first record of this nematode in the Western Hemisphere. Columbia root-knot nematode, $M$. chitwoodi, was found in a single golf course in California. Polymerase chain reaction restriction fragment length polymorphism of the intergenic region between the cytochrome oxidase and $16 S$ rRNA genes in the mitochondrial genome with restriction enzyme $S s p$ I was able to distinguish populations of $M$. graminis from $M$. marylandi, providing a fast and inexpensive method for future diagnosis of these nematodes from turf.
\end{abstract} report of M. marylandi in Arizona, California, Hawaii, Nevada, and
Turfgrass, in all its aspects, is a major industry in the United States. Considered as a "crop" and including commercial, residential, and recreational components, turf is the largest irrigated crop in America. Three times more irrigated turf than irrigated corn is grown, covering a surface area of more than $128,000 \mathrm{~km}^{2}(21)$. Golf is a primary consumer and producer of turf. The 16,000 golf courses in the United States utilize an estimated 908,000 ha of land, including 609,000 ha of maintained turfgrass (19). The economic impact of the golf course industry is significant, estimated in 2005 to have an annual value of $\$ 195$ billion (1).

Plant-parasitic nematodes are an important factor affecting the health, quality, production, and maintenance of turfgrass on golf courses. Nematodes frequently associated with turfgrass in the western United States include root-knot nematodes, Meloidogyne spp.; cyst nematodes, Heterodera spp.; the Pacific shoot gall nematode, Anguina pacificae; ring nematodes, Criconemella spp.; spiral nematodes, Helicotylenchus spp.; and stubby root nematodes, Trichodorus spp. Lance nematodes, Hoplolaimus spp., are seldom a problem in western states, and the sting nematode, Belonolaimus longicaudatus, considered the most damaging plant-parasitic nematode on turfgrasses in the Southeast (7), is on only a few isolated golf courses in the Coachella Valley of California. Recently, root-knot nematodes have gained attention as a serious threat to both cool- and warm-season turfgrasses (Fig. 1). In most cases, the species of root-knot nematodes found in western golf greens have not been identified. In 2002, 22 Southern California golf courses were surveyed to determine the range of genera and populations

Corresponding author: M. A. McClure, E-mail: mcclure@ag.arizona.edu

Mention of trade names or commercial products in this publication is solely for the purpose of providing specific information and does not imply recommendation or endorsement by the United States Department of Agriculture.

Accepted for publication 17 November 2011.

http://dx.doi.org/10.1094/PDIS-09-11-0808

(C) 2012 The American Phytopathological Society of plant-parasitic nematodes, including Meloidogyne spp. (34). Nematode counts were used in an attempt to determine the relationship between green quality and nematode populations but no significant correlation was found. More recently, a preliminary survey of nematodes in western golf greens included 17 courses in Arizona and 2 in California. In all, 12 of the 17 courses were infested with unidentified species of Meloidogyne and 3 of the 12 showed nematode counts above the "damage threshold", based on threshold calculations from Florida (13). A survey of 14 golf courses in Northern California found root-knot nematodes in $64 \%$ of the greens sampled but no attempt was made to determine the nematode species or to relate population levels to turf quality (40). A recent study examined the effect of root-knot nematode numbers on green performance for 18 bentgrass greens at the La Jolla Country Club in San Diego County, CA. No correlation was found between "good"- and "poor"-performing greens and nematode population levels, including those of an unidentified Meloidogyne sp. (33). Species identification is important for selection of appropriate turf grasses having resistance or tolerance to certain nematode species in new and renovated greens and for application of control measures that may be species specific, such as the introduction of Pasteuria penetrans for biological control.

The utility of molecular methods for identification of Meloidogyne spp. was demonstrated in a large-scale regional survey of potato acreage in the central United States (27). DNA markers that have aided identification of Meloidogyne spp. include the small subunit (SSU) 18S ribosomal RNA (rRNA) gene (25); the large subunit (LSU) 28S D2-D3 expansion segments of the rRNA gene $(4,23)$; the internal transcribed spacer (ITS) of rRNA gene (5); and the mitochondrial (mt)DNA marker, which spans the region between genes encoding cytochrome oxidase subunit II (COII) and $16 S$ rRNA $(3,26,32,41)$. In this article, we document the occurrence and distribution of root-knot nematodes in golf course greens in 10 western states, with identifications based on morphological examination and analysis of the D2-D3 expansion segments of 28S, partial 18S, ITS rRNA, and mtDNA gene sequences. A new diagnostic assay for Meloidogyne graminis and M. marylandi, based on restriction fragment length polymorphism (RFLP) of amplified mtDNA, is presented. 


\section{Materials and Methods}

Sampling. Samples consisting of soil and sod were collected from one or more putting greens of 238 golf courses. A large number of samples were provided by Frank P. Wong and Naveen Hyder at the Turf Pathology Diagnostic Laboratory, University of California, Riverside. Samples submitted to their laboratory were examined for other turf diseases and then forwarded to the University of Arizona for nematode assays. Additional samples were sent directly to the Arizona laboratory by golf course superintendents and pest control advisors. Samples from Utah and southern Arizona were collected by the authors. In all, 776 samples were processed. Samples sizes included 8- or 10-cm-diameter cup plugs and 8 to 12 bulked, 1.9-cm-diameter plugs taken to a depth of $7.5 \mathrm{~cm}$. Soil and roots were separated from the shoots and thatch. Meloidogyne infective juveniles (J2) were extracted from the soil and roots under an intermittent mist (2) for $72 \mathrm{~h}$ and the nematodes were collected from the tubes on a 500-mesh $(25-\mu \mathrm{m})$ sieve. Numbers of J2 per cubic centimeter of soil were determined by counting an aliquot of the extract, and individual specimens were hand picked for sequencing or fixation for light microscopy. Nematodes for sequencing were placed in DESS (43) and stored at $4^{\circ} \mathrm{C}$ for up to a year. Juveniles for light microscopy were fixed in cold $4 \%$ formalin and $1 \%$ glutaraldehyde in $0.01 \mathrm{M}$ phosphate buffer, $\mathrm{pH}$ 7.3., and stored at $4^{\circ} \mathrm{C}$.

Species identification. Topotypes of $M$. graminis were collected from the St. Augustine grass (Stenotaphrum secundatum) lawn around the Division of Plant Industry Laboratory in Winter Haven, FL, by Larry W. Duncan, University of Florida. Authenticated $M$.

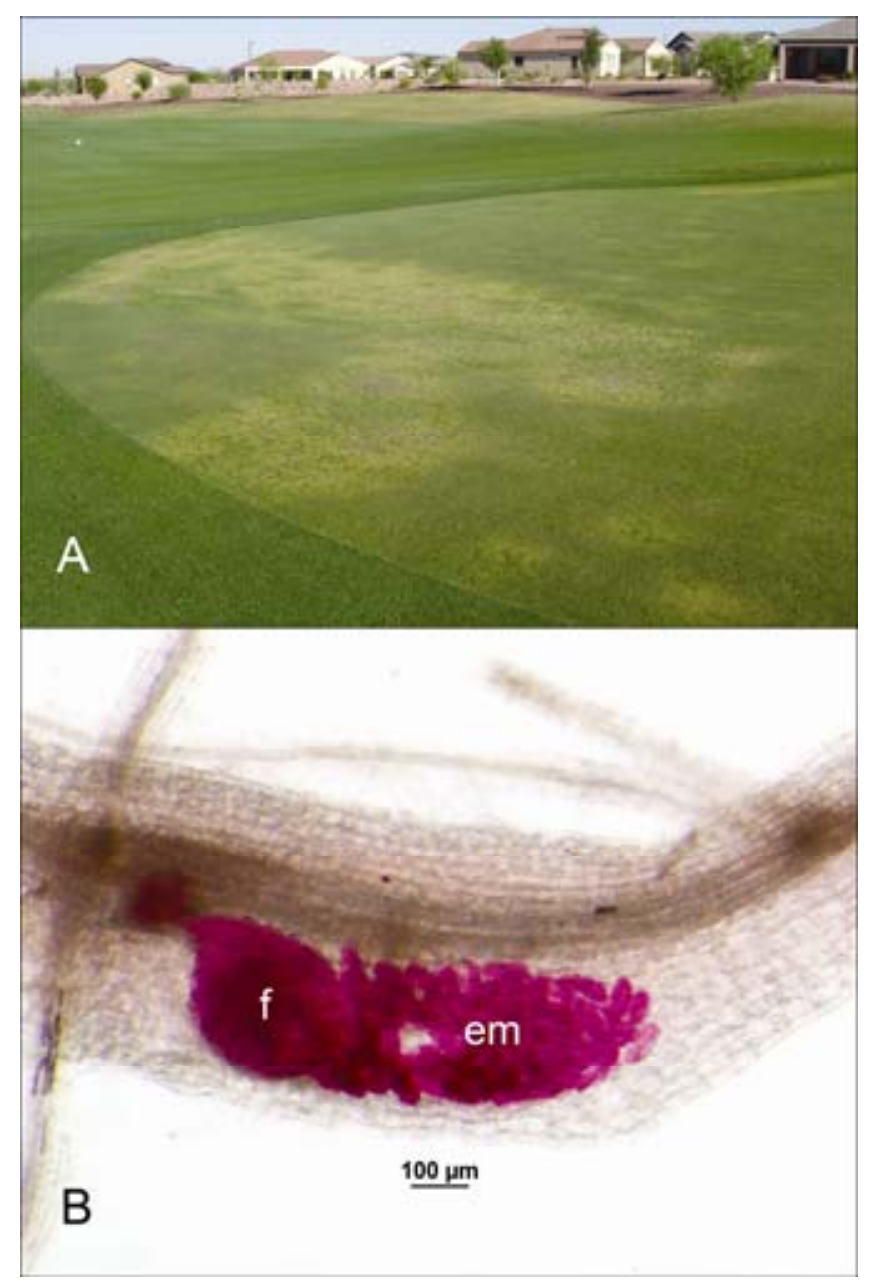

Fig. 1. Root-knot nematodes in turf grass. A, Damage caused by Meloidogyne marylandi to a bermudagrass (Cynodon dactylon) green; B, M. naasi female (f) with egg mass (em) in an annual bluegrass (Poa annua) root stained with acid fuchsin. minor and M. naasi from several locations in the United Kingdom were provided by Colin Fleming, Agri-Food and Biosciences Institute, Belfast, Northern Ireland, and M. graminicola was supplied by Teodora Cabasan at the International Rice Research Institute in the Philippines. James Starr, Texas A\&M University, contributed $M$. graminis and $M$. marylandi from Texas, and an Israeli population of M. marylandi came from the Division of Nematology, Institute of Plant Protection, ARO, the Volcani Center, Bet Dagan, Israel, courtesy of Yuji Oka. Topotypes of M. marylandi were collected by M. A. McClure from the collar surrounding the number 9 green at the University of Maryland Golf Course, College Park, MD (Table 1). Population number 500 from Brazos County, TX contained a mixture of M. graminis (GenBank JN241869 and JN241870) and $M$. marylandi (GenBank JN241838 and JN241868). The mixture was maintained on 'Jackpot' wheat in the greenhouse. Preliminary morphological identification of nematodes was made using $\mathbf{J} 2$ and female perineal patterns. Final species delimitation and identification was based on an integrated approach that considered morphological evaluation combined with molecular-based phylogenetic inference (tree-based methods) and sequence analyses (genetic distance methods) (30).

DNA extraction, polymerase chain reaction, cloning, and sequencing. Nematodes preserved in DESS were prepared for molecular analysis using two slightly different protocols. In protocol 1 (University of Arizona), nematodes were rinsed for $20 \mathrm{~min}$ in sterile distilled water and then transferred, individually, into a $10-\mu \mathrm{l}$ drop of sterile lysis buffer (10 mM Tris [pH 8.0], $0.25 \mathrm{M} \mathrm{GuHCl}$, $0.25 .0 \%$ Triton X-100, $0.25 \%$ Tween 20, and $2.0 \mu$ of Proteinase $\mathrm{K} ; 934$ units/ml) (11) on a clean glass cover slip and cut in half with a sharp scalpel blade. Scalpel blades were decontaminated prior to each cut by immersion in DNase Displace (Fisher Scientific, Pittsburgh) for several minutes, followed by three rinses in distilled water. The two halves of the nematode, and as much of the lysis buffer as possible, were transferred to a sterile, 0.6-ml polymerase chain reaction (PCR) tube containing $30 \mu \mathrm{l}$ of the same buffer. Lysis was completed by incubating the tubes at $60^{\circ} \mathrm{C}$ for 20 min followed by $10 \mathrm{~min}$ at $98^{\circ} \mathrm{C}$ to inactivate the Proteinase $\mathrm{K}$. When available, a minimum of eight $\mathrm{J} 2$ from each sample selected for analysis were lysed and at least three were sequenced. Lysed nematodes were stored at $-20^{\circ} \mathrm{C}$ for up to 2 weeks prior to PCR.

A Taq PCR Core kit (Qiagen, Valencia, CA) was used for PCR amplification of the D2-D3 region of the 28S gene and the ITS. Total reaction volume of $50 \mu \mathrm{l}$ contained $5 \mu \mathrm{l}$ of $10 \times$ PCR buffer, 5 $\mu \mathrm{l}$ of Q solution, $1 \mu \mathrm{l}$ of dNTPs, $1 \mu \mathrm{l}$ of primer F $(10 \mu \mathrm{mol}), 1 \mu \mathrm{l}$ of primer $\mathrm{R}(10 \mu \mathrm{mol}), 0.25 \mu \mathrm{l}$ of Taq, $31.75 \mu \mathrm{l}$ of nuclease-free water, and $5 \mu \mathrm{l}$ of DNA. Primers D2A (5'-ACAAGTACCGTGAGG GAAAGTTG-3') and D3B (5'-TCGGAAGGAACCAGCTACTA$\left.3^{\prime}\right)$ (18) were used for the D2-D3 region. For the ITS region, we used 5367 (5'-TTGATTACGTCCCTGCCCTTT-3') and F195 (5'TCCTCCGCTAAATGATATG-3') (18). The thermocycler was programmed as follows: $94^{\circ} \mathrm{C}$ for $3 \mathrm{~min}$; followed by 40 cycles of $94^{\circ} \mathrm{C}$ for $1 \mathrm{~min}, 52^{\circ} \mathrm{C}$ for $1 \mathrm{~min}$, and $72^{\circ} \mathrm{C}$ for $1 \mathrm{~min}$; with a final extension at $72^{\circ} \mathrm{C}$ for $10 \mathrm{~min}$. PCR products were separated on a $1.0 \%$ agarose gel, stained with ethidium bromide, and viewed under UV light. SCAR-PCR, using the primer sets and protocols described by Zijlstra (45), was used to identify $M$. chitwoodi and distinguish it from M. fallax. For sequencing, the PCR product bands were cut from the gel and purified using a QIAquick Gel Extraction Kit Gel (Qiagen). Purified bands were sequenced at the Genetics Core Facility at The University of Arizona. The sequences obtained were submitted for a search in GenBank using the BLASTN algorithm and compared with our sequences from authenticated populations.

In protocol 2 (United States Department of Agriculture, Beltsville, MD), specimens were mechanically disrupted in $20 \mu \mathrm{l}$ of extraction buffer (37), then stored in PCR tubes at $-80^{\circ} \mathrm{C}$ until needed. Extracts were prepared by incubating the tubes at $60^{\circ} \mathrm{C}$ for $60 \mathrm{~min}$ followed by $95^{\circ} \mathrm{C}$ for $15 \mathrm{~min}$ to deactivate the proteinase $\mathrm{K}$, and centrifuged briefly prior to use in PCR. Each $25-\mu \mathrm{l}$ PCR reaction contained 1 unit of Platinum Taq (Invitrogen, Carlsbad, CA), 
$1 \times$ reaction buffer $(20 \mathrm{mM}$ Tris- $\mathrm{HCl}[\mathrm{pH} 8.4], 50 \mathrm{mM} \mathrm{KCl}$, and 2.5 $\mathrm{mM} \mathrm{MgCl} 2$ ), $0.2 \mathrm{mM}$ dNTP mix, $0.3 \mu \mathrm{M}$ each primer, and $2 \mu \mathrm{l}$ of nematode extract. Partial ( $3^{\prime}$ end) $18 \mathrm{~S}$ sequence was amplified from selected survey populations using primer $18 \mathrm{~s} 1.2\left(5^{\prime}\right.$ GGCGATCAGATACCGCCCTAGTT-3') with 18sr2b (5'-TAC AAAGGGCAGGGACGTAAT-3'). Cycling conditions were 1 cycle of $94^{\circ} \mathrm{C}$ for $2 \mathrm{~min}$; followed by 40 cycles of $94^{\circ} \mathrm{C}$ for $20 \mathrm{~s}$, $59^{\circ} \mathrm{C}$ for $30 \mathrm{~s}$, and $72^{\circ} \mathrm{C}$ for $30 \mathrm{~s}$; and finishing with 1 cycle of $72^{\circ} \mathrm{C}$ for $5 \mathrm{~min}$. A longer $18 \mathrm{~S}$ sequence was obtained from reference populations by generating two additional overlapping PCR fragments. Reactions with primers 988F (5'-CTCAAAGAT TAAGCCATGC-3') and 1912R (5'-TTTACGGTCAGAACT AGGG-3') were amplified as above, substituting annealing at $65^{\circ} \mathrm{C}$; reactions with primers 550F (5'-GGCAAGTCTGGTGCC AGCAGCC-3') and 1108R (5'-CCACTCCTGGTGGTGCCC TTCC-3') were amplified as described by Holterman et al. (12). For some populations, the ITS 1 and 2 rDNA region was amplified with primers TW81 (5'-GGTCAATGTTCAGAAATTTGTGG-3') and AB28 (5'-GGTCAATGTTCAGAAATTTGTGG-3') according to Skantar et al. (31); 28S D2-D3 rDNA was amplified as described previously $(9,42)$. Amplification of the mtDNA region between the COII and $16 S$ rRNA genes included primers 1RNAF (5'TACCTTTGACCAATCACGCT-3') and COIIR (5'-GGTCAATGT TCAGAAATTTGTGG- $3^{\prime}$ ). Cycling conditions included 1 cycle at $94^{\circ} \mathrm{C}$ for $2 \mathrm{~min}$; followed by 45 cycles of $94^{\circ} \mathrm{C}$ for $30 \mathrm{~s}, 48^{\circ} \mathrm{C}$ for $30 \mathrm{~s}$, and $68^{\circ} \mathrm{C}$ for $2 \mathrm{~min}$; ending with 1 cycle of $68^{\circ} \mathrm{C}$ for $5 \mathrm{~min}$. PCR products were analyzed by electrophoresis on $1 \%$ agarose and $1 \times$ sodium borate-EDTA (SB). Gels were stained with ethidium bromide and visualized using the U:Genius gel documentation system (Syngene, Frederick, MD). DNA was excised from the gels and purified as described in protocol 1. PCR products were quantified using a Nanodrop 8000 spectrophotometer (Thermo Fisher Scientific, Pittsburgh) and sequenced at the University of Maryland
Center for Biosystems Research. Selected amplicons were cloned with the Strataclone PCR Cloning Kit (Stratagene, La Jolla, CA) according to the manufacturer's instructions. Plasmid DNA was prepared with the QiaPrep Spin Miniprep Kit and digested with EcoRI to verify the presence of the insert. Two or more clones per amplicon were sequenced. DNA sequences were assembled using Sequencher 4.10.1 (Genecodes, Ann Arbor, MI) and analyzed using the BLASTN megablast program optimized for highly similar sequences (http://www.ncbi.nlm.nih.gov/blast/Blast.cgi). Selected sequences were submitted to GenBank (Tables 1 and 2).

Mitochondrial PCR products from selected reference and golf course populations were used for analysis of restriction fragment polymorphisms. PCR product ( 8 to $10 \mu \mathrm{l}$, approximately $600 \mathrm{ng}$ ) was digested overnight in $20-\mu$ reactions with restriction enzymes DraI or $S s p$ I in the supplied reaction buffers (New England Biolabs, Ipswich, MA). Digested DNA was run on a $2.5 \%$ agarose gel buffered with SB, stained, and photographed as described above. The length of each restriction fragment was determined by virtual digestion of mitochondrial sequences in Sequencher.

Sequence and phylogenetic analysis. The newly obtained sequences for each gene were aligned using ClustalX 1.83 (16) with default parameters with corresponding published gene sequences $(8,24,27,36,38)$. Outgroup taxa for each dataset were chosen according to the results of previously published data $(8,27,36)$. Phylogenetic analyses of the dataset were performed with Bayesian inference (BI) using MrBayes 3.1.2 (14). BI analysis under the GTR + I + G model was initiated with a random starting tree and was run with four chains for $1.0 \times 10^{6}$ generations. The Markov chains were sampled at intervals of 100 generations. Two runs were performed for each analysis. The log-likelihood values of the sample points stabilized after approximately $10^{3}$ generations. The topologies were used to generate a $50 \%$ majority rule consensus tree. Posterior probabilities (PPs) are given on appropriate clades.

Table 1. Reference populations used for identification of Meloidogyne spp. in a survey of golf course greens in the western United States

\begin{tabular}{|c|c|c|c|c|c|c|}
\hline \multirow[b]{2}{*}{ Species } & \multirow[b]{2}{*}{ Source } & \multirow[b]{2}{*}{ Origin } & \multicolumn{4}{|c|}{ GenBank accession number } \\
\hline & & & D2-D3 of $28 S$ & ITS-rRNA $^{\mathrm{a}}$ & $18 S$ & MtDNA \\
\hline Meloidogyne chitwoodi & $\begin{array}{l}\text { Russell Ingham: Oregon State University, } \\
\text { Corvallis } \\
\text { Kathy Merrifield: Oregon State University, } \\
\text { Corvallis } \\
\text { Saad Hafez: University of Idaho, Parma }\end{array}$ & $\begin{array}{l}\text { Oregon } \\
\text { Washington } \\
\text { Idaho }\end{array}$ & $\begin{array}{l}\cdots \\
\cdots \\
\cdots\end{array}$ & $\begin{array}{l}\text { JN157868 } \\
\text { JN241864, } \\
\text { JN241865 }\end{array}$ & $\cdots$ & $\begin{array}{l}\text { JN241945- } \\
\text { JN241949 } \\
\text { JN241902 }\end{array}$ \\
\hline M. fallax & $\begin{array}{l}\text { Hans Helder: Wageningen University, } \\
\text { The Netherlands } \\
\text { Lieven Waeyenberge: Instituut voor } \\
\text { Landbouw-en Visserijonderzoek, Belgium }\end{array}$ & $\begin{array}{l}\text { The Netherlands } \\
\text { The Netherlands }\end{array}$ & $\begin{array}{l}\text { JN157869 } \\
\text { JN157848 }\end{array}$ & $\ldots$ & $\begin{array}{l}\text { JN389789 } \\
\text { JN389788 }\end{array}$ & $\begin{array}{l}\text { JN241954 } \\
\text { JN241950- } \\
\text { JN241953 }\end{array}$ \\
\hline M. graminicola & $\begin{array}{l}\text { Teodora Cabasan: International Rice Research } \\
\text { Institute, Los Banos, The Philippines } \\
\text { George Abawi: Cornell University, } \\
\text { Ithaca, NY } \\
\text { George Abawi: Cornell University, } \\
\text { Ithaca, NY }\end{array}$ & $\begin{array}{l}\text { The Philippines } \\
\text { Bangladesh } \\
\text { India }\end{array}$ & $\begin{array}{l}\text { JN157844 } \\
\ldots\end{array}$ & $\begin{array}{l}\text { JN241866, } \\
\text { JN241867 }\end{array}$ & $\cdots$ & $\begin{array}{l}\text { JN241927, } \\
\text { JN241929 } \\
\text { JN241926, } \\
\text { JN241939 }\end{array}$ \\
\hline M. graminis & $\begin{array}{l}\text { Larry Duncan, University of Florida, } \\
\text { Lake Alfred } \\
\text { Nicholas Sekora: University of Florida, } \\
\text { Gainesville }\end{array}$ & $\begin{array}{l}\text { Type locality, } \\
\text { Florida } \\
\text { Florida }\end{array}$ & $\begin{array}{l}\text { JN157849 } \\
\text { JN157850 }\end{array}$ & $\cdots$ & JN389786 & $\begin{array}{l}\text { JN241922- } \\
\text { JN241925 }\end{array}$ \\
\hline M. marylandi & $\begin{array}{l}\text { Michael McClure: University of Arizona, Tucson } \\
\text { Yuji Oka: The Volcani Institute, Gilat Research } \\
\text { Center, Negev, Israel }\end{array}$ & $\begin{array}{l}\text { Type locality, } \\
\text { Maryland } \\
\text { Israel }\end{array}$ & $\begin{array}{l}\text { JN157851 } \\
\text { JN157852 }\end{array}$ & $\cdots$ & $\begin{array}{l}\cdots \\
\text { JN241856 }\end{array}$ & $\begin{array}{l}\text { JN241917, } \\
\text { JN241955 } \\
\text { JN241918- } \\
\text { JN241921 }\end{array}$ \\
\hline M. minor & $\begin{array}{l}\text { Colin Fleming: Agri-Food and Biosciences } \\
\text { Institute, Belfast, UK }\end{array}$ & United Kingdom & JN157846 & JN157871 & $\begin{array}{l}\text { JN389787, } \\
\text { JN241839, } \\
\text { JN241840 }\end{array}$ & 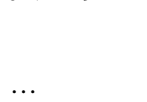 \\
\hline & $\begin{array}{l}\text { Lieven Waeyenberge: Instituut voor } \\
\text { Landbouw-en Visserijonderzoek, Belgium }\end{array}$ & Belgium & $\begin{array}{l}\text { JN628436, } \\
\text { JN628437 }\end{array}$ & $\ldots$ & $\ldots$ & $\ldots$ \\
\hline M. naasi & $\begin{array}{l}\text { Colin Fleming: Agri-Food and Biosciences } \\
\text { Institute, Belfast, UK } \\
\text { Kathy Merrifield: Oregon State University, } \\
\text { Corvallis } \\
\text { Washington }\end{array}$ & United Kingdom & JN157847 & $\begin{array}{l}\ldots \\
\ldots\end{array}$ & $\begin{array}{l}\text { JN241841 } \\
\ldots \\
\text { JN241909 }\end{array}$ & JN241944 \\
\hline
\end{tabular}

a ITS = internal transcribed spacer. 
Sequence differences between samples were calculated with PAUP* $4 \mathrm{~b} 10$ (35) as an absolute distance matrix and the percentage was adjusted for missing data.

\section{Results}

Root-knot nematode distribution. Of the 238 golf courses sampled in this survey, $60 \%$ were infested with root-knot nematodes. Numbers of $\mathrm{J} 2$ ranged from 0.08 to 53.3 per cubic centime- ter of soil (data not shown). In all, 112 of the infested samples were selected for sequencing of DNA markers amplified from J2 DNA: 24 from Arizona, 58 from California, 5 from Hawaii, 4 from Nevada, 4 from Oregon, 4 from Utah, and 13 from Washington (Table 2). The most common species was M. naasi, (found in $52 \%$ of the samples), which was distributed from San Diego County in Southern California to King County in Washington (Fig. 2). One sample from Washoe County, NV also contained M. naasi. None

Table 2. Meloidogyne spp. collected in a survey of golf course greens in the western United States

\begin{tabular}{|c|c|c|c|c|c|c|c|}
\hline \multirow[b]{2}{*}{ Species } & \multirow[b]{2}{*}{ Pop. ${ }^{a}$} & \multicolumn{2}{|c|}{ Origin } & \multicolumn{4}{|c|}{ GenBank accession number } \\
\hline & & County & State & D2-D3 of $28 S$ & ITS-rRNA $^{b}$ & $18 S$ & MtDNA \\
\hline Meloidogyne chitwoodi & 186 & San Luis Obispo & California & JN019321 & $\ldots$ & JN632480 & $\ldots$ \\
\hline \multirow[t]{14}{*}{ M. graminis } & 027 & Maricopa & Arizona & .. & JN157864; & & \\
\hline & & & & & $\begin{array}{l}\text { N241882- } \\
\text { JN241885 }\end{array}$ & JN241851 & JN241901 \\
\hline & 268 & Pinal & Arizona & JN019326 & $\ldots$ & JN241837 & JN241907 \\
\hline & 182 & Kern & California & JN019327 & $\ldots$ & JN241854 & JN241915 \\
\hline & 428 & Orange & California & JN019328 & $\ldots$ & $\ldots$ & $\ldots$ \\
\hline & 090 & Riverside & California & JN019329 & JN241860- & & \\
\hline & & & & & N241863 & JN241843 & JN241900 \\
\hline & 699 & Riverside & California & $\ldots$ & JN157865 & $\ldots$ & $\ldots$ \\
\hline & 108 & San Diego & California & JN019330 & & $\ldots$ & $\ldots$ \\
\hline & 724 & San Diego & California & $\ldots$ & JN241857- & & \\
\hline & & & & & N241859 & JN241834 & JN241898 \\
\hline & 730 & San Diego & California & JN019331 & $\ldots$ & $\ldots$ & $\ldots$ \\
\hline & 474 & Kona & Hawaii & JN019339 & $\ldots$ & $\ldots$ & $\ldots$ \\
\hline & 281 & Clark & Nevada & JN019332 & JN157866 & & \\
\hline \multirow[t]{40}{*}{ M. marylandi } & 001 & Maricopa & Arizona & JN019333 & $\begin{array}{l}\text { JN157853; } \\
\text { JN241874- } \\
\text { N241877 }\end{array}$ & JN241848 & JN241916 \\
\hline & 017 & Maricopa & Arizona & JN019334 & JN157854 & JN241844 & JN241904 \\
\hline & 019 & Maricopa & Arizona & JN019335 & $\ldots$ & $\ldots$ & $\ldots$ \\
\hline & 021 & Maricopa & Arizona & JN019336 & $\ldots$ & $\ldots$ & $\ldots$ \\
\hline & 034 & Maricopa & Arizona & JN019337 & $\ldots$ & JN241852 & JN241905 \\
\hline & 389 & Maricopa & Arizona & $\cdots$ & $\begin{array}{l}\text { JN241878- } \\
\text { N241881 }\end{array}$ & JN241850 & JN241906 \\
\hline & 472 & Maricopa & Arizona & JN019338 & $\ldots$ & $\ldots$ & $\ldots$ \\
\hline & 623 & Maricopa & Arizona & JN019340 & JN157855 & $\ldots$ & $\ldots$ \\
\hline & 333 & Pima & Arizona & JN019341 & $\begin{array}{l}\text { JN157856; } \\
\text { JN241890- } \\
\text { N241896 }\end{array}$ & JN241833 & JN241908 \\
\hline & 494 & Pima & Arizona & JN019342 & $\ldots$ & $\ldots$ & $\ldots$ \\
\hline & 603 & Pima & Arizona & JN019343 & $\ldots$ & $\ldots$ & $\ldots$ \\
\hline & 618 & Pima & Arizona & JN019344 & JN157857 & $\ldots$ & $\ldots$ \\
\hline & 625 & Pima & Arizona & JN019345 & $\ldots$ & $\ldots$ & $\ldots$ \\
\hline & 627 & Pima & Arizona & JN019346 & $\ldots$ & $\ldots$ & $\ldots$ \\
\hline & 637 & Pima & Arizona & JN019347 & $\ldots$ & $\ldots$ & $\ldots$ \\
\hline & 641 & Pima & Arizona & JN019348 & $\ldots$ & $\ldots$ & $\ldots$ \\
\hline & 643 & Pima & Arizona & JN019325 & $\ldots$ & $\ldots$ & $\ldots$ \\
\hline & 656 & Pima & Arizona & JN019349 & JN157858 & $\ldots$ & $\ldots$ \\
\hline & 658 & Pima & Arizona & JN019350 & $\ldots$ & $\ldots$ & $\ldots$ \\
\hline & 721 & Pima & Arizona & JN019351 & $\ldots$ & $\ldots$ & $\ldots$ \\
\hline & 047 & Pinal & Arizona & JN019352 & $\ldots$ & $\ldots$ & $\ldots$ \\
\hline & 330 & Pinal & Arizona & JN019353 & $\ldots$ & $\ldots$ & $\ldots$ \\
\hline & 650 & Inyo & California & JN019354 & $\ldots$ & $\ldots$ & $\ldots$ \\
\hline & 256 & Los Angeles & California & JN019355 & $\ldots$ & $\ldots$ & $\ldots$ \\
\hline & 427 & Orange & California & JN019356 & $\ldots$ & $\ldots$ & $\ldots$ \\
\hline & 480 & Orange & California & JN019357 & $\ldots$ & $\ldots$ & $\ldots$ \\
\hline & 481 & Orange & California & JN019358 & $\ldots$ & $\ldots$ & $\ldots$ \\
\hline & 359 & Riverside & California & JN019359 & $\ldots$ & $\ldots$ & $\ldots$ \\
\hline & 373 & Riverside & California & JN019360 & $\ldots$ & $\ldots$ & $\ldots$ \\
\hline & 434 & Riverside & California & JN019361 & $\ldots$ & $\ldots$ & $\ldots$ \\
\hline & 071 & San Bernardino & California & JN019362 & $\ldots$ & $\ldots$ & $\ldots$ \\
\hline & 436 & Hawaii & Hawaii & JN019363 & $\ldots$ & $\ldots$ & $\ldots$ \\
\hline & 447 & Kona & Hawaii & JN019364 & $\ldots$ & $\ldots$ & $\ldots$ \\
\hline & 474 & Kona & Hawaii & JN019365 & $\ldots$ & $\ldots$ & $\ldots$ \\
\hline & 463 & Maui & Hawaii & JN019366 & $\ldots$ & $\ldots$ & $\ldots$ \\
\hline & 386 & Clark & Nevada & JN019367 & $\ldots$ & $\ldots$ & $\ldots$ \\
\hline & 473 & Clark & Nevada & JN019368 & $\ldots$ & $\ldots$ & $\ldots$ \\
\hline & 737 & Washington & Utah & JN019369 & $\ldots$ & $\ldots$ & $\ldots$ \\
\hline & 763 & Washington & Utah & JN157845 & $\ldots$ & $\ldots$ & $\ldots$ \\
\hline & & & & & & $(c$ & d on next page) \\
\hline
\end{tabular}

a Population number.

b ITS = internal transcribed spacer. 
were found in samples from Arizona, Colorado, or Hawaii. In all, 38 of the samples (34\%) contained $\mathrm{J} 2$ whose sequences matched those of $M$. marylandi and 11 samples matched the sequences of M. graminis. Three samples from Washington contained $M$. minor, a species previously not known in North America. M. chitwoodi was recovered from a golf course in San Luis Obispo County, CA. $M$. graminicola was not found in any of the samples sequenced.
Seven samples from Colorado, five from Montana, and three from Idaho were examined but none contained root-knot nematodes.

Species identification using molecular approaches. Phylogenetic relationships among the root-knot nematodes, as inferred from BI analysis of the 28S D2-D3 expansion segments, ITS rRNA, partial 18S rRNA, and mtDNA gene sequences, are given in Figures 3, 4, 5, and 6, respectively. Using traditional morphologi-

Table 2. (continued from preceding page)

\begin{tabular}{|c|c|c|c|c|c|c|c|}
\hline \multirow[b]{2}{*}{ Species } & \multirow[b]{2}{*}{ Pop. $^{a}$} & \multicolumn{2}{|c|}{ Origin } & \multicolumn{4}{|c|}{ GenBank accession number } \\
\hline & & County & State & D2-D3 of $28 S$ & ITS-rRNA $^{b}$ & $18 S$ & MtDNA \\
\hline \multirow[t]{3}{*}{ M. minor } & 383 & Snohomish & Washington & JN019322 & JN157867 & JN389792 & $\ldots$ \\
\hline & 437 & King & Washington & JN019323 & $\ldots$ & JN389791 & $\ldots$ \\
\hline & 438 & King & Washington & JN019324 & $\ldots$ & $\ldots$ & $\ldots$ \\
\hline \multirow[t]{60}{*}{ M. naasi } & 179 & Alameda & California & JN019265 & $\ldots$ & $\ldots$ & $\ldots$ \\
\hline & 209 & Alameda & California & JN019266 & $\ldots$ & $\ldots$ & $\ldots$ \\
\hline & 452 & Alameda & California & JN019267 & $\ldots$ & $\ldots$ & $\ldots$ \\
\hline & 151 & Contra Costa & California & JN019268 & $\ldots$ & $\ldots$ & $\ldots$ \\
\hline & 068 & Los Angeles & California & JN019269 & $\ldots$ & $\ldots$ & $\ldots$ \\
\hline & 115 & Los Angeles & California & JN019270 & $\ldots$ & $\ldots$ & $\ldots$ \\
\hline & 248 & Los Angeles & California & JN019271 & $\ldots$ & $\ldots$ & $\ldots$ \\
\hline & 267 & Los Angeles & California & JN019272 & $\ldots$ & $\ldots$ & $\ldots$ \\
\hline & 195 & Marin & California & JN019273 & $\ldots$ & $\ldots$ & $\ldots$ \\
\hline & 009 & Monterey & California & JN019274 & $\ldots$ & $\ldots$ & $\ldots$ \\
\hline & 010 & Monterey & California & JN019275 & JN157859; & & \\
\hline & & & & & $\begin{array}{l}\text { JN241871- } \\
\text { N241873 }\end{array}$ & JN241842 & JN241897 \\
\hline & 023 & Monterey & California & JN019276 & $\ldots$ & $\ldots$ & $\ldots$ \\
\hline & 025 & Monterey & California & JN019277 & $\ldots$ & $\ldots$ & $\ldots$ \\
\hline & 311 & Monterey & California & JN019278 & JN157860 & $\ldots$ & $\ldots$ \\
\hline & 713 & Monterey & California & JN019279 & $\ldots$ & $\ldots$ & $\ldots$ \\
\hline & 734 & Monterey & California & JN019280 & JN157861 & $\ldots$ & $\ldots$ \\
\hline & 191 & Orange & California & JN019281 & $\ldots$ & $\ldots$ & $\ldots$ \\
\hline & 264 & Orange & California & JN019282 & $\ldots$ & $\ldots$ & $\ldots$ \\
\hline & 342 & Orange & California & JN019283 & $\ldots$ & $\ldots$ & $\ldots$ \\
\hline & 138 & Riverside & California & JN019284 & $\ldots$ & $\ldots$ & $\ldots$ \\
\hline & 098 & Sacramento & California & JN019285 & $\ldots$ & $\ldots$ & $\ldots$ \\
\hline & 277 & San Bernardino & California & JN019286 & $\ldots$ & $\ldots$ & $\ldots$ \\
\hline & 726 & San Bernardino & California & JN019287 & $\ldots$ & $\ldots$ & $\ldots$ \\
\hline & 163 & San Diego & California & JN019288 & $\ldots$ & JN241847 & JN241913 \\
\hline & 255 & San Diego & California & JN019289 & $\ldots$ & $\ldots$ & $\ldots$ \\
\hline & 730 & San Diego & California & JN019290 & $\ldots$ & $\ldots$ & $\ldots$ \\
\hline & 670 & San Francisco & California & JN019291 & JN157862 & $\ldots$ & $\ldots$ \\
\hline & 263 & San Luis Obispo & California & JN019292 & $\ldots$ & JN241846 & JN241910 \\
\hline & 162 & San Mateo & California & JN019293 & $\ldots$ & $\ldots$ & $\ldots$ \\
\hline & 663 & San Mateo & California & JN019294 & $\ldots$ & $\ldots$ & $\ldots$ \\
\hline & 074 & Santa Barbara & California & JN019295 & $\ldots$ & $\ldots$ & $\ldots$ \\
\hline & 599 & Santa Barbara & California & JN019296 & $\ldots$ & $\ldots$ & $\ldots$ \\
\hline & 101 & Santa Clara & California & JN019297 & $\ldots$ & $\ldots$ & $\ldots$ \\
\hline & 147 & Santa Clara & California & JN019298 & $\ldots$ & $\ldots$ & $\ldots$ \\
\hline & 218 & Santa Clara & California & JN019299 & $\ldots$ & $\ldots$ & $\ldots$ \\
\hline & 245 & Santa Clara & California & JN019300 & $\ldots$ & JN241836 & JN241912 \\
\hline & 422 & Santa Clara & California & JN019301 & $\ldots$ & $\ldots$ & $\ldots$ \\
\hline & 057 & Stanislaus & California & JN019302 & $\ldots$ & $\ldots$ & $\ldots$ \\
\hline & 707 & Stanislaus & California & & JN241886- & & \\
\hline & & & & & N241889 & JN241853 & JN241899 \\
\hline & 282 & Ventura & California & & $\ldots$ & JN241849 & JN241911 \\
\hline & 041 & Ventura & California & JN019303 & $\ldots$ & $\ldots$ & $\ldots$ \\
\hline & 013 & Washoe & Nevada & JN019304 & $\ldots$ & $\ldots$ & $\ldots$ \\
\hline & 410 & Jackson & Oregon & JN019305 & $\ldots$ & $\ldots$ & $\ldots$ \\
\hline & 054 & Lane & Oregon & JN019306 & $\ldots$ & JN241845 & JN241903 \\
\hline & 512 & Multnomah & Oregon & JN019307 & $\ldots$ & $\ldots$ & $\ldots$ \\
\hline & 275 & Washington & Oregon & JN019308 & $\ldots$ & $\ldots$ & $\ldots$ \\
\hline & 547 & Washington & Utah & JN019309 & $\ldots$ & $\ldots$ & $\ldots$ \\
\hline & 591 & Utah & Utah & JN019310 & JN157863 & $\ldots$ & $\ldots$ \\
\hline & 530 & Clackamas & Washington & JN019311 & $\ldots$ & $\ldots$ & $\ldots$ \\
\hline & 194 & King & Washington & JN019312 & $\ldots$ & JN241855 & JN241914 \\
\hline & 437 & King & Washington & JN019313 & $\ldots$ & $\ldots$ & $\ldots$ \\
\hline & 438 & King & Washington & JN019314 & $\ldots$ & $\ldots$ & $\ldots$ \\
\hline & 524 & King & Washington & JN019315 & $\ldots$ & $\ldots$ & $\ldots$ \\
\hline & 351 & Kitsap & Washington & JN019316 & $\ldots$ & $\ldots$ & $\ldots$ \\
\hline & 361 & Pierce & Washington & JN019317 & $\ldots$ & $\ldots$ & $\ldots$ \\
\hline & 578 & Pierce & Washington & JN019318 & $\ldots$ & $\ldots$ & $\ldots$ \\
\hline & 150 & Snohomish & Washington & JN019319 & $\ldots$ & $\ldots$ & $\ldots$ \\
\hline & 424 & Snohomish & Washington & JN019320 & $\ldots$ & $\ldots$ & $\ldots$ \\
\hline
\end{tabular}


cal taxonomic characteristics and molecular criteria (apomorphies and genetic distances), we distinguished the following species within studied samples from our survey: $M$. naasi, $M$. minor, $M$. chitwoodi, M. marylandi, and M. graminis.

In all, 148 sequences, 115 of which were new, were included in the analysis of D2-D3 expansion segments of the 28S rRNA gene. The $28 \mathrm{~S}$ rRNA alignment was $721 \mathrm{bp}$ in length. Several moderate and highly supported major clades were distinguished in the majority consensus BI tree (Fig. 3): (i) $M$. naasi + M. graminicola $(\mathrm{PP}=$ 100), (ii) $M$. exigua $(\mathrm{PP}=100)$, (iii) $M$. minor $+M$. chitwoodi + M. fallax $(\mathrm{PP}=100)$, (iv) M. hapla + M. dunensis + M. silvestris + M. hispanica + Meloidogyne sp. from the tropical group $(\mathrm{PP}=94)$, and (v) M. marylandi $+M$. graminis $(\mathrm{PP}=94)$. Sequences of $M$. marylandi formed two subclades. Intraspecific sequence variation for species reached the following percentages: $M$. naasi, $0.7 \%$ (5 bp); M. minor, $0.5 \%$ (3 bp); M. chitwoodi, $0.3 \%$ (2 bp); M. fallax, 0.4\% (3 bp); M. marylandi, 1.7\% (9 bp); M. graminis, $1.0 \%$ (7 bp); and $M$. graminicola, $0.1 \%$ ( $1 \mathrm{bp})$. Interspecific sequence variation between some species pairs was $4.6 \%$ (27 bp) for $M$. marylandi and $M$. graminis and $0.4 \%$ ( $3 \mathrm{bp}$ ) $M$. chitwoodi and $M$. fallax.

The ITS rRNA alignment was 679 bp in length and included 99 sequences, 57 of which were newly obtained in this study. The following moderate and highly supported major clades were distinguished in the ITS tree (Fig. 4): (i) M. naasi + M. graminicola (PP = 100), (ii) $M$. chitwoodi and M. fallax ( $\mathrm{PP}=97)$, (iii) $M$. minor $(\mathrm{PP}=100)$, (iv) $M$. marylandi $+M$. graminis $(\mathrm{PP}=100),(\mathrm{v}) M$. silvestris, (vi) M. hapla, (vii) M. dunensis, (viii) $M$. hispanica $+M$. enterolobii + Meloidogyne sp. from the tropical group, and (ix) $M$. panyuensis. Intraspecific sequence variation for some species was as follows: M. naasi, 0 to $1.5 \%$ (0 to $9 \mathrm{bp}$ ), M. minor, 0 to $1 \%$ (0 to $3 \mathrm{bp}$ ), M. chitwoodi, 0 to $0.4 \%$ (0 to $2 \mathrm{bp}$ ); M. fallax, 0 to $0.2 \%$ (0 to 2 nucleotides); $M$. marylandi, 0 to $6.5 \%$ (0 to $35 \mathrm{bp}$ ), $M$. graminis, 0 to $4.3 \%$ ( 0 to $23 \mathrm{bp}$ ), and $M$. graminicola, 0 to $0.9 \%$ (0 to $5 \mathrm{bp}$ ). Interspecific sequence variation between $M$. chitwoodi and M. fallax was 1.7 to $2.0 \%$ ( 8 to $11 \mathrm{bp}$ ).

In all, 102 sequences, 30 of which were new, were included in the $18 \mathrm{~S}$ rRNA alignment with a length of $656 \mathrm{bp}$. M. graminis and $M$. marylandi clustered together (Fig. 5) and were not distinguishable. $M$. chitwoodi and $M$. fallax also had similar sequences, and they differed from closely related M. naasi by one nucleotide.

The mtDNA alignment included 71 sequences, was 462 bp long, and included 45 novel mtDNA sequences. The BI tree (Fig. 6)

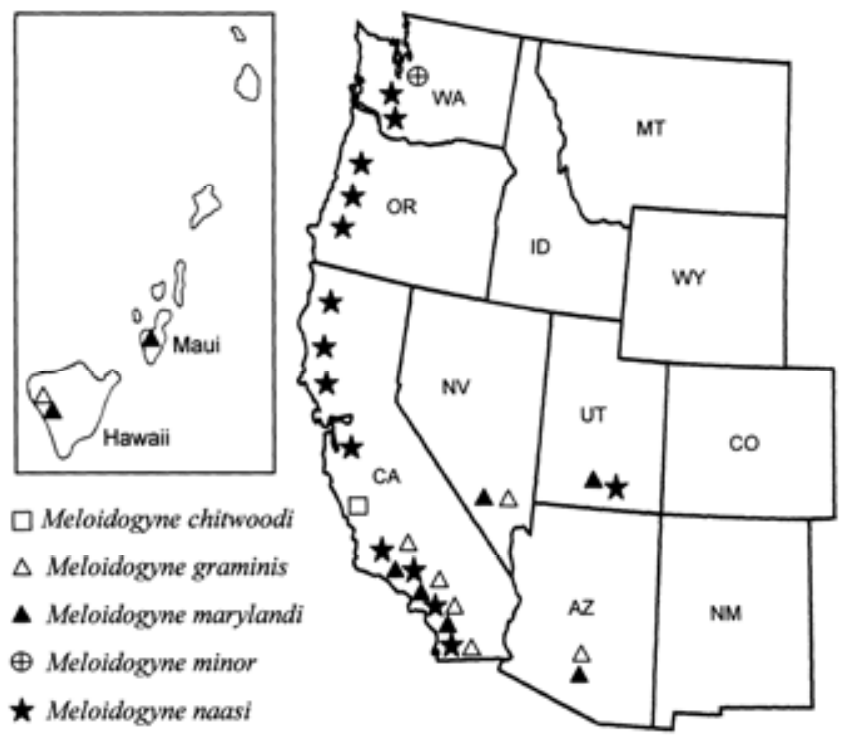

Fig. 2. Distribution of root-knot nematodes in golf course greens in the Western United States. Seven samples from Colorado, five from Montana, and three from Idaho were negative for Meloidogyne spp. Wyoming and New Mexico were not included in the survey. Symbols represent distribution of the species. contained several highly supported major clades ( $\mathrm{PP}=99$ to 100): (i) $M$. chitwoodi + M. fallax, (ii) $M$. naasi $+M$. graminicola, (iii) M. marylandi + M. graminis, (iv) M. hapla + M. partityla, and (v) M. enterolobii. Sequences for $M$. graminis, $M$. graminicola, and $M$. naasi each formed two subclades. Intraspecific sequence divergence for some species were varied: across the whole $M$. graminis clade, divergence was 0 to $5 \%$ ( 0 to $22 \mathrm{bp}$ ) whereas, within same subclade, it was 0 to $0.6 \%$ ( 0 to $3 \mathrm{bp}$ ); $M$. marylandi variation was 0 to $2.0 \%$ ( 0 to $9 \mathrm{bp}$ ), with a maximum differences between Maryland and other isolates; $M$. naasi variation was 0 to $0.9 \%$ (0 to 4 bp). M. chitwoodi and M. fallax differed by one nucleotide deletion or insertion.

Mitochondrial PCR products from the root-knot nematodes included in the study ranged in size from 487 to $539 \mathrm{bp}$. Sequences were extremely AT-rich $(>78 \%)$, limiting the restriction enzymes predicted to generate diagnostically informative polymorphisms. Digestion of the mitochondrial PCR products with restriction enzyme $S s p$ I allowed discrimination among several of the species found in the survey (Table 3; Fig. 7). Identical restriction patterns of $M$. chitwoodi and $M$. fallax were distinct from $M$. naasi and the other species but not each other. The pattern of $M$. graminis differed from that of $M$. marylandi. A unique restriction pattern was also found for M. graminicola.

To further test discrimination between $M$. graminis and $M$. marylandi, RFLP analysis was conducted on multiple individuals from reference populations from Florida and Israel, respectively, and from the type locality of M. marylandi, a golf course in College Park, MD. Distinct restriction patterns for each species were observed with enzymes DraI and SspI, with all J2 tested from a population giving the same pattern (Fig. 8A and $\mathrm{B}$, respectively). Digestion of PCR products generated from $\mathrm{J} 2$ of selected golf course isolates gave mixed results with these enzymes. DraI digestion of mtDNA products amplified from one or more $\mathrm{J} 2$ from $M$. graminis survey population numbers $027,090,182$, and 724 revealed the presence of a 227-bp fragment, similar to the largest fragment from $M$. marylandi, rather than the 306-bp band observed for Florida M. graminis (Fig. 9A). Two of four J2 tested from Texas population number 500 (Fig 9B, lanes 1 and 3) also showed patterns that matched M. marylandi (lane 15). SspI digests of the PCR products from the same specimens clearly separated $M$. graminis from $M$. marylandi (Fig. 9B). The $S s p \mathrm{I}$ profiles of juveniles from survey population numbers $027,090,182,724$, and 500 conformed to the Florida $M$. graminis pattern.

In silico analysis of mtDNA sequences from the reference populations, golf course isolates, and sequences available from GenBank confirmed the restriction site variability seen in the RFLP gel patterns (Table 3). Sequence types corresponding to different DraI restriction profiles were found for $M$. graminis populations; the addition of two DraI sites changed a 307-bp fragment to 227 $+78 \mathrm{bp}$, and changed a 74-bp fragment to $57+16 \mathrm{bp}$. Two sequence types that could lead to DraI and $S s p \mathrm{I}$ polymorphisms were also found for $M$. graminicola. No differences affecting these enzymes were found in $M$. naasi, M. chitwoodi, or $M$. fallax sequences.

M. graminis and M. marylandi sequences were further examined in silico for the utility of $A l u \mathrm{I}, \mathrm{Dde \textrm {I }}$, and $\mathrm{Pac \textrm {I }}$ restriction sites. Two $A l u \mathrm{I}$ restriction sites were found in mtDNA sequences of $M$. graminis survey populations but not in the reference population from Florida. The same two sites were found in sequences from the M. marylandi type locality (Maryland) but were not present in the population from Israel; nor were they found in any of the survey populations identified as $M$. marylandi. Due to the intraspecific variation affecting these sites, $A l u \mathrm{I}$ cannot discriminate $M$. graminis from $M$. marylandi. The enzyme DdeI cleaves $M$. graminis mtDNA into two fragments but does not cut $M$. marylandi, although the fragment length differences may be too slight to be practically useful. A single PacI site was found in all $M$. graminis and $M$. marylandi sequences. Thus, this enzyme cannot distinguish these species but may be useful for setting them apart from others. 


\section{Discussion}

Molecular identification of root-knot nematodes. During the present survey, we identified five species of root-knot nematodes:
M. naasi, M. minor, M. chitwoodi, M. marylandi, and M. graminis. With the exception of $M$. naasi, which has a distinctively attenuated tail, the four other species of Meloidogyne encountered in this survey are not easily diagnosed by morphology of the $\mathrm{J} 2$, the stage

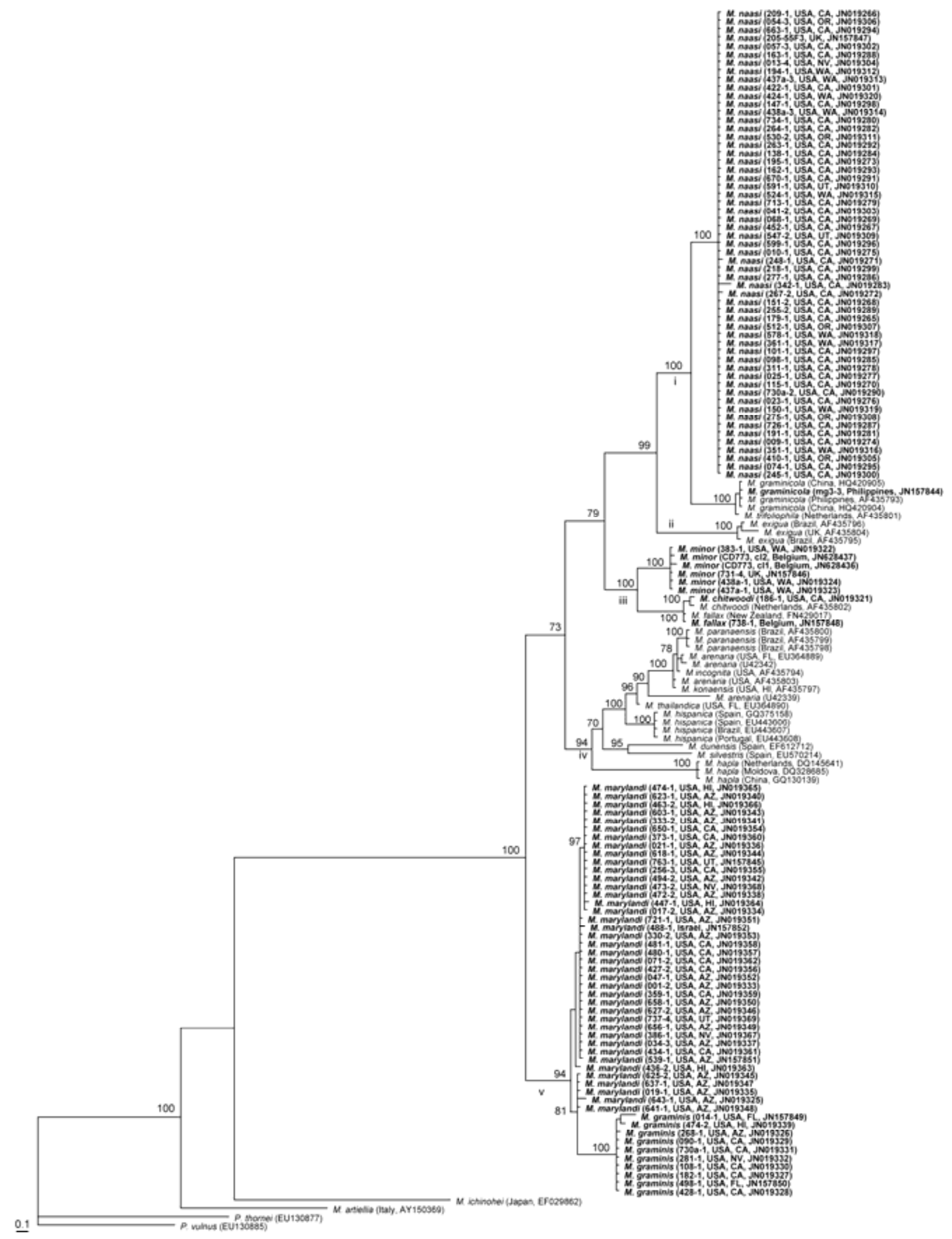

Fig. 3. Phylogenetic relationships within root-knot nematodes as inferred from Bayesian analysis of the D2-D3 of $28 \mathrm{~S}$ rRNA gene sequences. Posterior probability values more than $70 \%$ are given on appropriate clades. Newly obtained sequences are indicated in bold. 
routinely found in soil samples. In recent years, analysis of DNA sequences has been increasingly used for identification of Meloidogyne spp. (25). In the present study, we used sequence information from three fragments of rRNA genes and one fragment of
mtDNA for sample identification. For the first time, sequences are reported here for D2-D3 of 28S rRNA from M. naasi, M. minor, and $M$. marylandi; for mtDNA from $M$. marylandi and $M$. naasi; for ITS rRNA from $M$. graminis and $M$. marylandi; and for partial

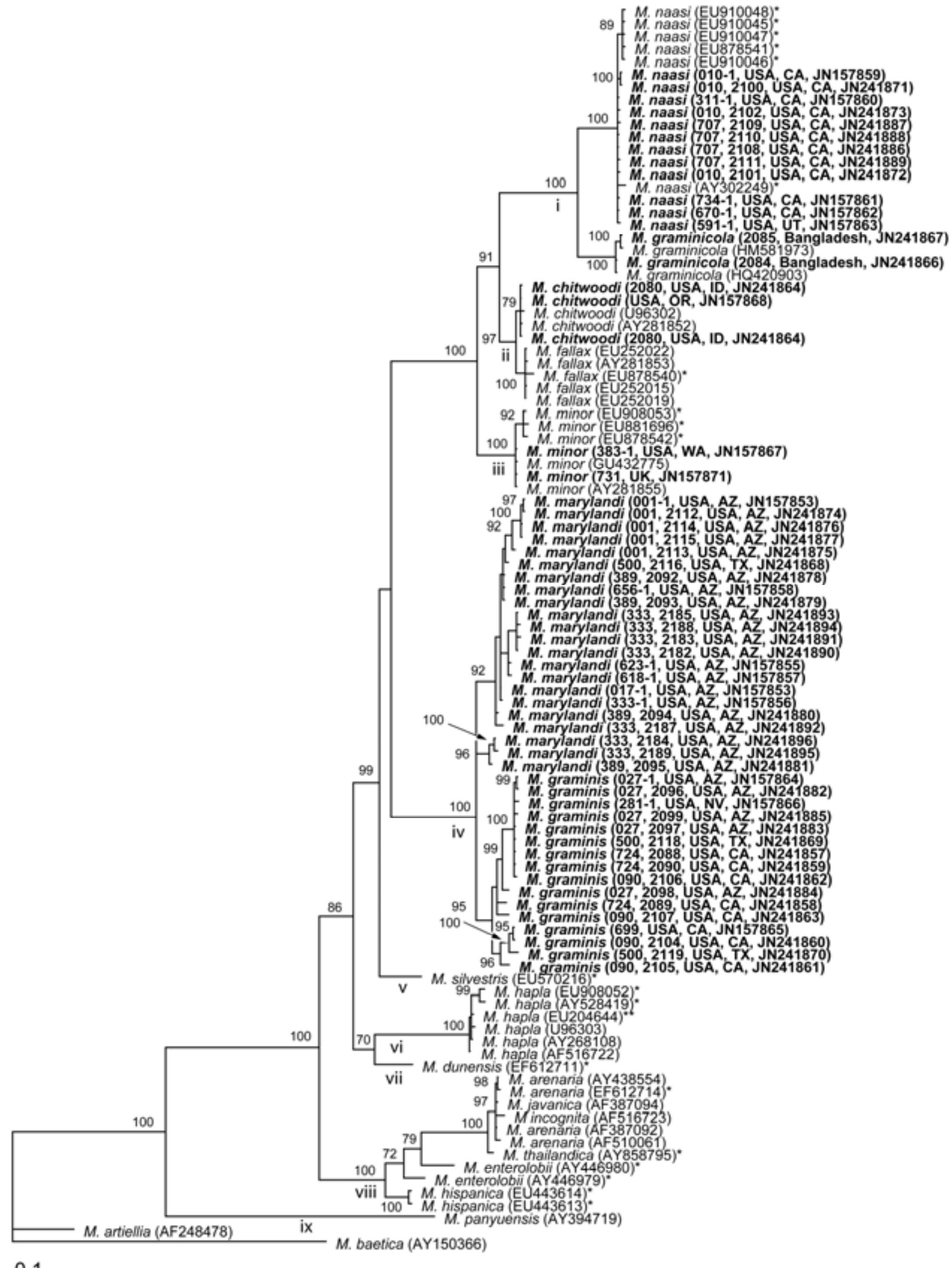

\section{$\underline{0.1}$}

Fig. 4. Phylogenetic relationships within root-knot nematodes as inferred from Bayesian analysis of the internal transcribed spacer (ITS)1-5.8S rRNA-ITS2 gene sequences. Posterior probability values more than $70 \%$ are given on appropriate clades. Newly obtained sequences are indicated in bold; * indicates only ITS1 used for the analysis; ${ }^{* *}$ indicates originally identified as Meloidogyne ethiopica. 
18S rRNA gene from $M$. marylandi. These gene fragments varied with respect to their usefulness for species diagnostics. The D2-D3 expansion segments of $28 \mathrm{~S}$ rRNA and the mtDNA sequences showed the best discrimination power. Although the ITS rRNA gene sequences distinguished most root-knot nematode species from each other, heterogeneity in this gene fragment did not allow unambiguous diagnosis of $M$. graminis and M. marylandi. Conversely, the partial $3^{\prime}$ end of $18 \mathrm{~S}$ rRNA was relatively conservative and did not contain enough nucleotide differences to separate $M$. graminis from M. marylandi or M. chitwoodi from M. fallax.

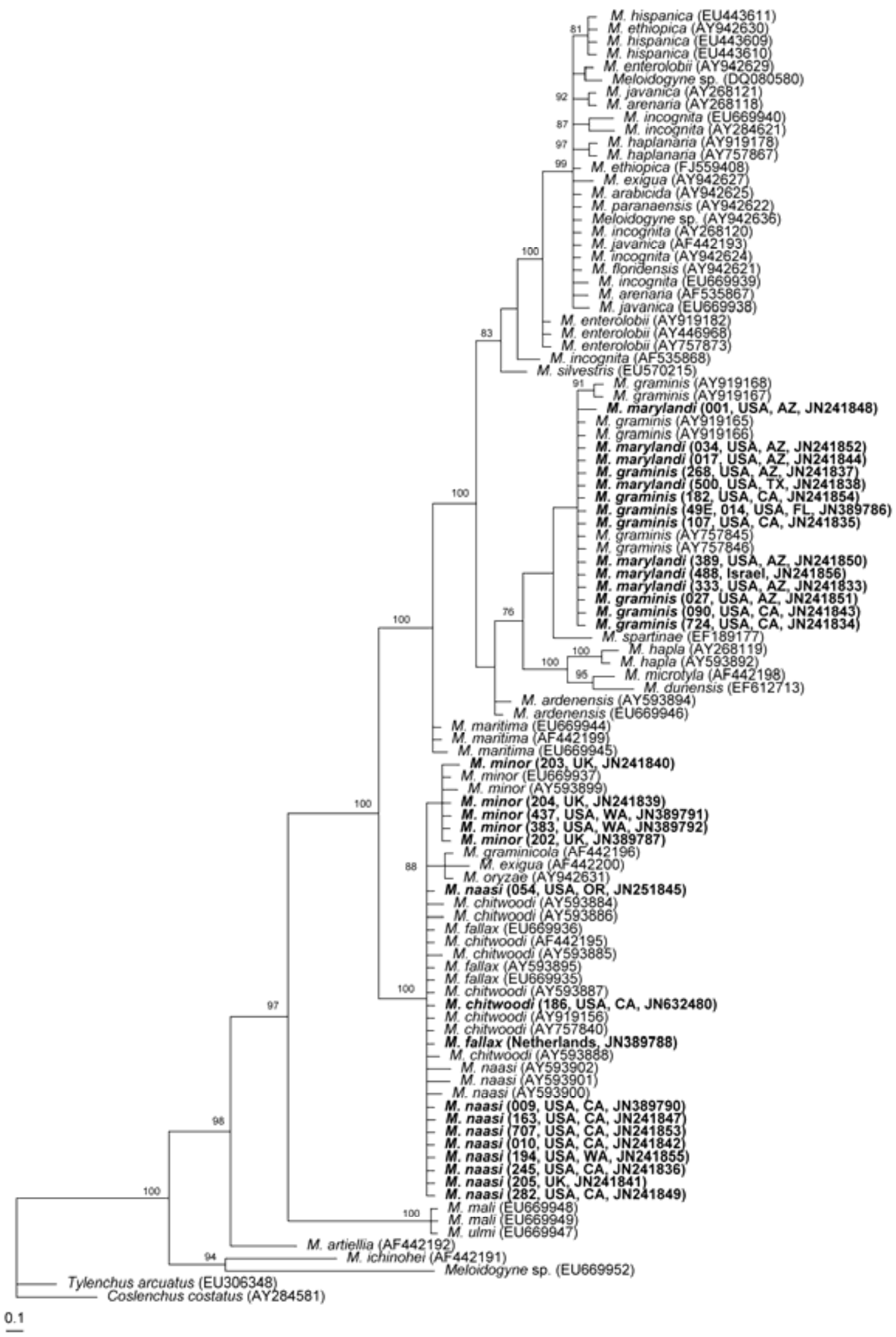

Fig. 5. Phylogenetic relationships within root-knot nematodes as inferred from Bayesian analysis of the partial 18S rRNA gene sequences. Posterior probability values more than $70 \%$ are given on appropriate clades. Newly obtained sequences are indicated in bold. 


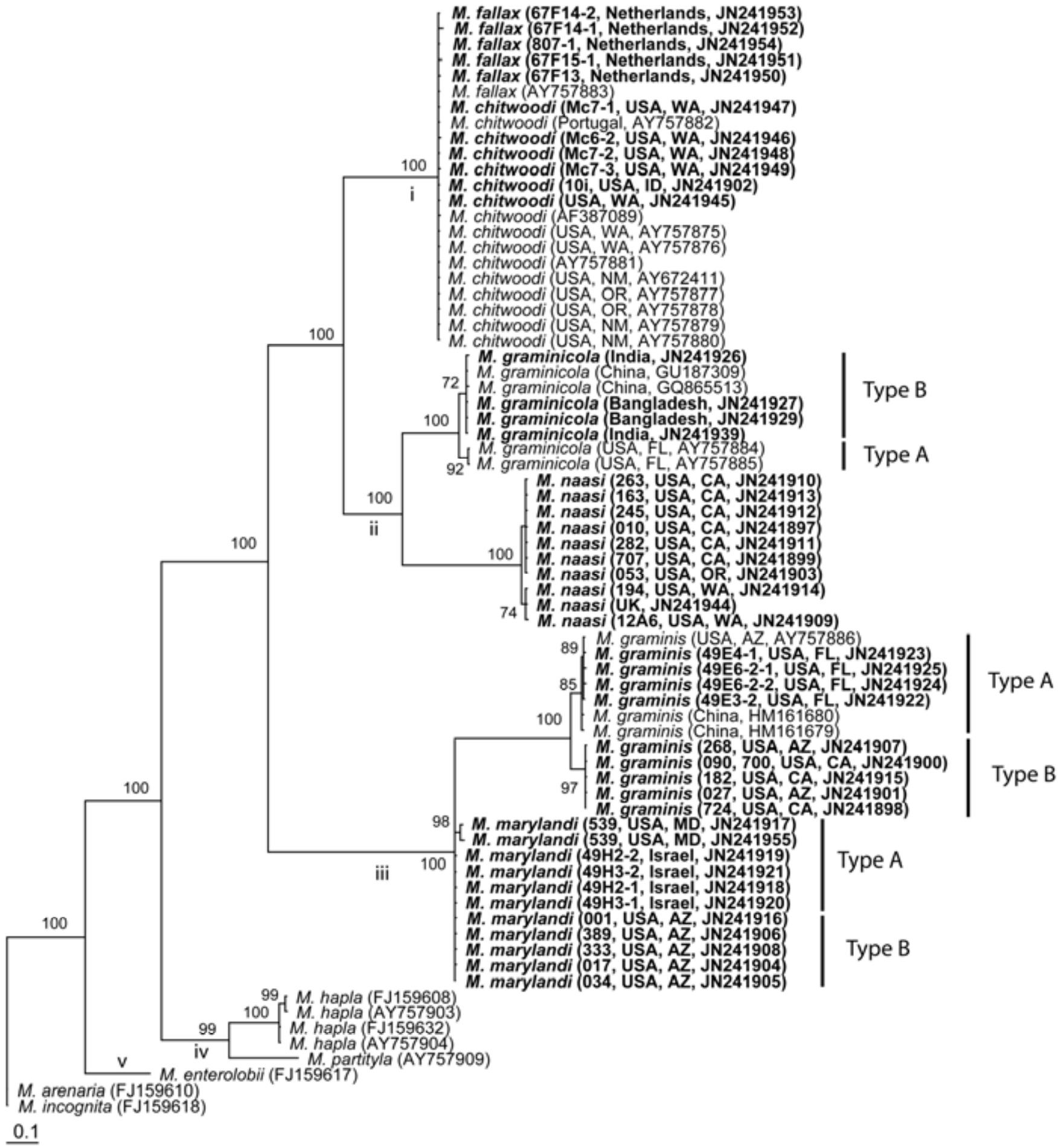

Fig. 6. Phylogenetic relationships within root-knot nematodes as inferred from Bayesian analysis of the partial mitochondrial DNA sequences. Posterior probability values more than $70 \%$ are given on appropriate clades. Newly obtained sequences are indicated in bold.

Particular attention has been given to the region of mtDNA including partial COII and $16 \mathrm{~S}$ rRNA genes, owing to the rapid evolution of this molecule relative to nuclear rRNA genes (15). This marker was originally targeted as a potential means for differentiating the five common Meloidogyne spp. by giving differentsized amplified PCR products $(3,18,22,25,28)$. A large group of species, including $M$. hapla, M. chitwoodi, M. fallax, M. graminicola, M. graminis, M. mali, M. marylandi, M. microtyla, M. naasi, M. oryzae, M. suginamiensis, and M. trifoliophila, fell into the smallest size class, those lacking an AT-rich region in the amplified product (2). Our analysis also revealed higher interspecific sequence variation in mtDNA compared with nuclear rRNA genes in all studied root-knot nematodes, except for $M$. chitwoodi and $M$. fallax, whose mtDNA fragment surprisingly differed by a single deletion or insertion.

Our sequence analysis of mtDNA also showed the presence of different haplotypes for some species (Table 3), although only for $M$. graminicola did there appear to be any association of sequence type with geographic origin (Asia versus the United States). Powers et al. (26) previously reported three mtDNA haplotypes for $M$. chitwoodi. Sequences obtained for $M$. chitwoodi populations in our study conformed to those designated type A, including populations from Washington, Idaho, and Portugal, but distinct from type $\mathrm{C}$ populations from Oregon and New Mexico. M. graminis sequences 
Table 3. Approximate sizes of restriction fragments generated by two diagnostic enzymes after digestion of mitochondrial DNA polymerase chain reaction products from root-knot nematodes, Meloidogyne spp.

\begin{tabular}{|c|c|c|c|c|}
\hline \multirow[b]{2}{*}{ Species } & \multirow[b]{2}{*}{ Origin } & \multicolumn{3}{|c|}{ Size (bp) } \\
\hline & & Length & DraI & SspI \\
\hline Meloidogyne graminicola type A & Florida & 531 & 311,220 & $114,111,102,92,65,47$ \\
\hline M. graminicola type B & Bangladesh, China, India & 531 & $313,156,62$ & $167,113,112,92,47$ \\
\hline M. graminis type A & Arizona, Florida, China & 540 & $307,74,42,33,32,30,22$ & $187,148,115,61,28$ \\
\hline M. graminis type B & Arizona, California, Texas & 537 & $227,78,57,41,33,33,30,22,16$ & $187,147,114,60,28$ \\
\hline M. marylandi type A & Arizona, Maryland, Israel & 534 & $228,80,78,62,39,33,14$ & $148,122,115,89,60$ \\
\hline M. marylandi type B & Arizona & 534 & $228,78,62,50,39,33,30,14$ & $148,122,115,89,60$ \\
\hline M. fallax & The Netherlands & 520 & $258,118,86,40,18$ & $239,234,47$ \\
\hline M. chitwoodi & Oregon & 519 & $257,118,86,40,18$ & $238,234,47$ \\
\hline M. naasi & United Kingdom & 530 & $312,127,91$ & $225,93,77,50,39,30,16$ \\
\hline
\end{tabular}

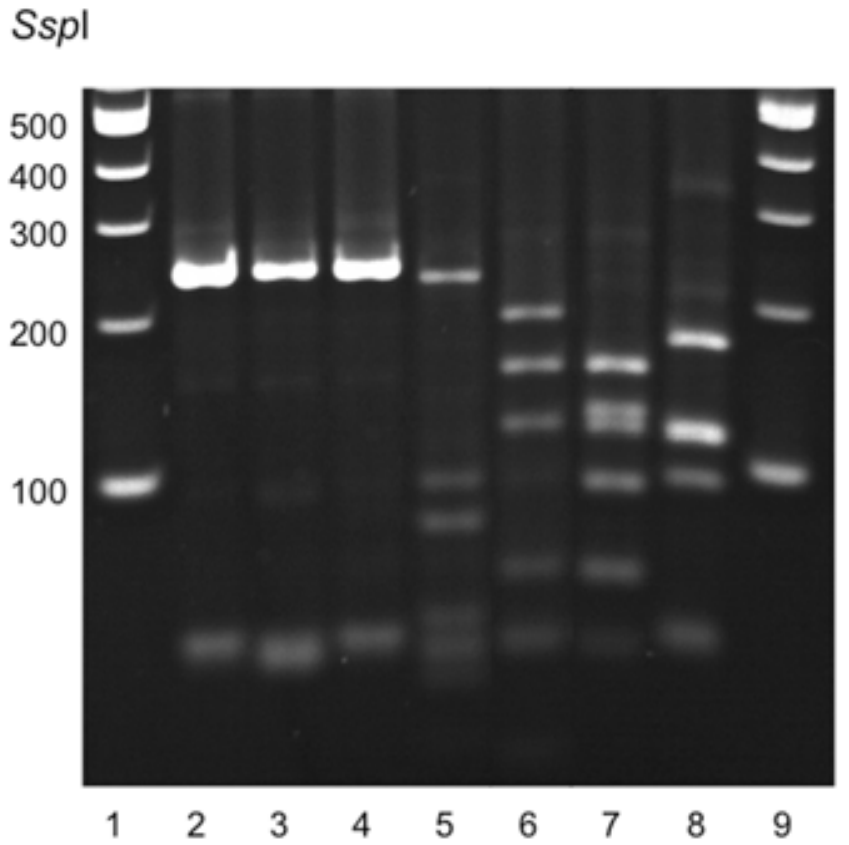

Fig. 7. Polymerase chain reaction restriction fragment length polymorphism profile of mitochondrial DNA for root-knot nematode species. Lanes 1 and 9: 100-bp DNA ladder; lane 2: Meloidogyne chitwoodi, Washington, United States; lane 3: M. fallax, The Netherlands; lane 4: M. fallax, The Netherlands; lane 5: M. naasi, (205), United Kingdom; lane 6: M. graminis, (014), Florida, United States; lane 7: M. marylandi, (488), Israel; lane 8: M. graminicola, India.

from the Florida reference population corresponded to type A sequences reported from Arizona (AY757886) and China (HM161679 and HM161680), whereas those from California (numbers 090, 182, and 724) and Arizona (numbers 027 and 268) constituted a novel type B pattern not reported previously. No other $M$. marylandi mtDNA sequences were available for comparison; therefore, there is need for additional sampling to determine the extent of sequence types present in other geographic areas.

PCR-RFLP of mtDNA has been a reliable and rapid method of diagnostics for the root-knot nematodes $(25,26)$. We found that amplification of the mtDNA fragment followed by digestion with SspI consistently discriminated populations of M. graminis from $M$. marylandi and was not subject to the heterogeneity that can confound DraI RFLPs. To our knowledge, this is the first diagnostic assay designed to separate these two species, providing a simple, inexpensive assay that can be applied to DNA derived from single juveniles. However, due to the low complexity and high AT content of mtDNA in root-knot nematodes, further confirmation by sequencing multiple DNA markers may be necessary for identification of new or unusual populations.

Root-knot nematodes from golf courses. Except for Florida, where the incidence is $89 \%$ (6), the percentage of golf courses in this survey that were infested with root-knot nematodes (60\%) was considerably higher than that reported from elsewhere in North

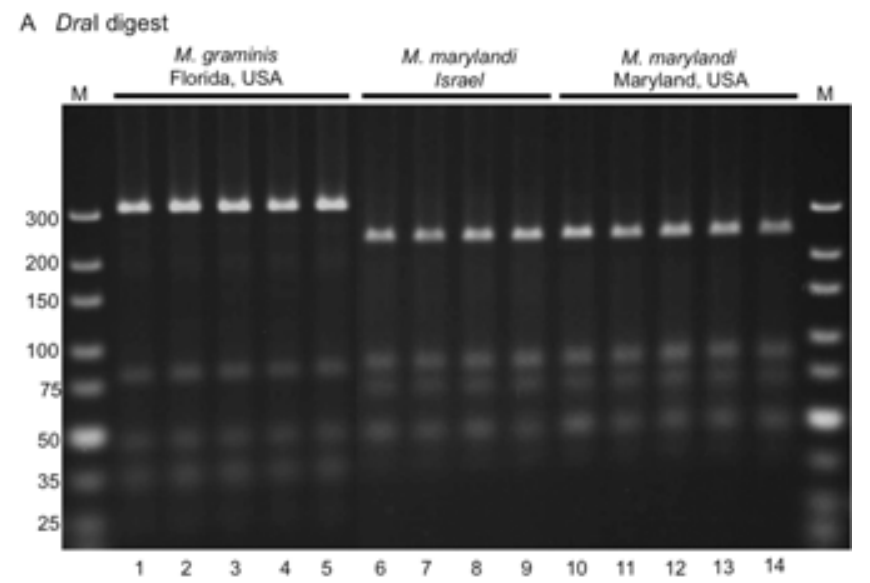

B Sspl digest

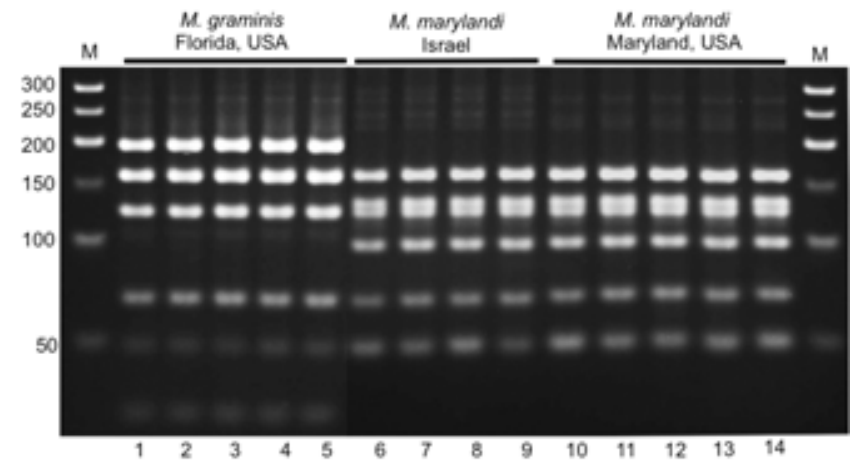

Fig. 8. Restriction enzyme profiles of mitochondrial DNA polymerase chain reaction products generated from selected root-knot nematode reference populations and digested with enzymes A, Dral or B, Sspl. Infective juveniles from reference populations served as template in A and B. Lanes 1-5, Meloidogyne graminis, Lake Alfred, FL; lanes 6-9: M. marylandi, Israel; lanes 10-14: M. marylandi, College Park, MD; lanes M = 100-bp ladder.

America: Alabama, 36.8\% (29); Ontario, Canada, 6.6\% (44); and Kansas, $0.0 \%$ (39). These differences could be due to a variety of reasons, including edaphic, climatic, and procedural factors, such as sampling methods. One explanation for an increase in the incidence of root-knot nematodes in recent years is the trend for golf course greens to be constructed or renovated according to standards set by the United States Golf Association, which specifies a sand content of $92 \%$ or greater in the top 12 inches. The sand content of most greens is further augmented by frequent top dressings with sand, (up to $15 \mathrm{~kg}$ of sand per square meter annually), providing an ideal substrate for root-knot nematodes to infect susceptible hosts. In general, the distribution of Meloidogyne spp. on Western golf courses was correlated with average annual temperatures: $M$. naasi, $M$. minor, and $M$. chitwoodi in the cooler regions and $M$. marylandi and $M$. graminis in the warmer regions. M. marylandi 
was found in golf course greens in Death Valley, CA, at $65 \mathrm{~m}$ below sea level, the lowest elevation in the United States, and one of the hottest and driest. The type of turf grown in these regions may also influence distribution of the species. Golf greens in cooler climates are commonly annual bluegrass (Poа апnua) or bentgrass (Agrostis spp.), whereas those in the warmer climates are either bentgrass or bermudagrass (Cynodon dactylon). Fescue greens (Festuca spp.) and others such as Zoysia spp. are not widely used in the Western states. Precise determination of the host was difficult, primarily because many of the greens sampled consisted of mixed turf. Bentgrass greens in coastal California, Oregon, Utah, and Washington frequently are invaded by $P$. аnnua, which may constitute $50 \%$ or more of the turf, and bermudagrass is a common invader of bentgrass greens in parts of Southern California and Arizona.

M. graminis is one of the most common root-knot nematodes on turf grasses in the southern United States, especially Florida (20). It is less common in the Southwest, where the principal species in golf course greens is M. marylandi. In the current survey, both species were found cohabiting a single green on a golf course in San Diego County, CA. Whether this resulted from a coincidence of preferred climatic and edaphic factors was not resolved but, clearly, the opportunity exists for $M$. graminis to be more widely distributed than presently found. Morphologically, the J2 are very similar and, where $M$. marylandi predominates, mixed populations would be very difficult to detect by microscopic examination alone. However, RFLP analysis of multiple individuals using SspI could be used to screen for the presence of both species.

$M$. chitwoodi is widely distributed on potato and other crops in the Pacific Northwest, including Northern California, but its occurrence on a mature golf course green near the central coast of California was unexpected. The host range of M. chitwoodi includes both monocots and dicots $(10,22,28)$ but, to our knowledge, this is the first report of a turfgrass host in a golf course green, in

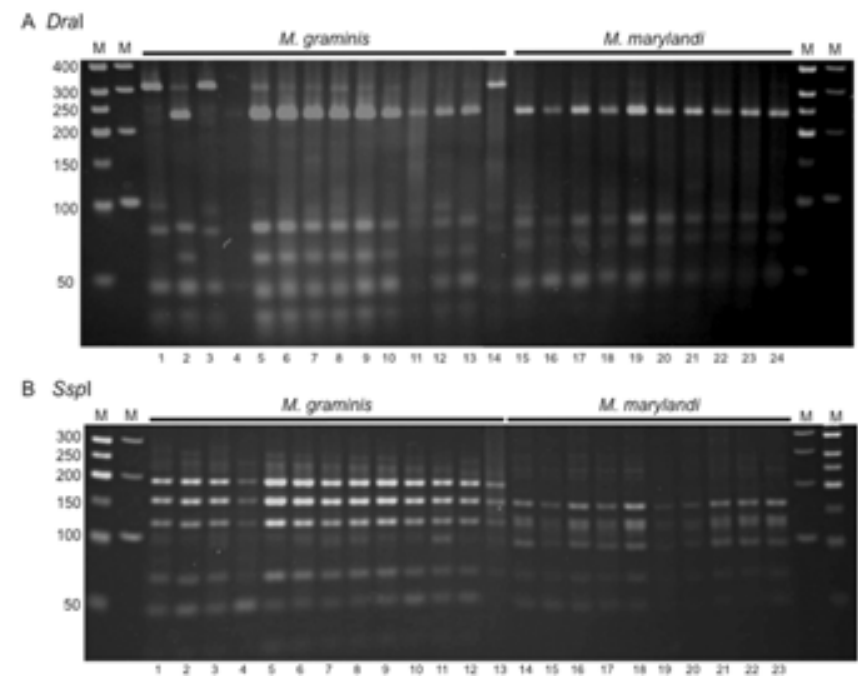

Fig. 9. Restriction enzyme profiles of mitochondrial DNA polymerase chain reaction products generated from selected root-knot nematode golf course populations and digested with enzymes A, Dral or B, Sspl. A, Meloidogyne graminis survey populations include lanes 1-4: number 500; lanes 5 and 6: number 724; lanes 7 and 8: number 027; lanes 9 and 10: number 090; lane 11: number 268; lanes 12 and 13: number 182: lane 14: reference population, Lake Alfred, FL. M. marylandi populations include lane 15: reference population, Israel; lanes 16 and 17: number 333; lanes 18 and 19: number 389; lane 20: number 001; lanes 21 and 22: number 017; lanes 23 and 24: number 034. B, M. graminis survey populations include lanes 1-4: number 500; lane 5: number 724; lanes 6 and 7: number 027 ; lanes 8 and 9 : number 090; lane 10: number 268; lanes 11 and 12: number 182: lane 13: reference population, Lake Alfred, FL. M. marylandi populations include lane 14: reference population, Israel; lanes 15 and 16: number 333; lanes 17 and 18: number 389; lane 19: number 001; lanes 20 and 21: number 017; lanes 22 and 23: number 034 . Lanes $M=100-b p$ ladder. Further details for populations can be found in Tables 1 and 2. this case, a mixture of $P$. апnиa and creeping bentgrass $(A$. palustris).

M. minor is a relatively new species that was first found on potato in The Netherlands in 2004 and, subsequently, on turfgrass from a score of golf courses and sports fields in Ireland and the United Kingdom, where it causes symptoms of a yellow patch disease (17). Recently it has been found in Portugal (C. Fleming, personal communication) and Chile (G. Karssen, personal communication), raising interesting questions regarding its likely origin. The two golf courses in Washington where $M$. minor was detected in our survey are within $50 \mathrm{~km}$ of each other. Other courses nearby may also be infested but a dedicated survey would be required to determine the distribution of $M$. minor in the Pacific Northwest. In the United Kingdom, mixed populations of M. minor and $M$. naasi are common. In the Washington golf courses, these species occurred together in a ratio of approximately 1:4. The potential exists for $M$. minor to spread from these two golf courses to agricultural crops but the risk has not been assessed. Both of the golf courses in Washington where M. minor was found are relatively isolated from commercial agriculture. Spread to other golf courses in the region is more likely. Golf clubs and golf shoes, contaminated with soil containing $M$. minor eggs or $\mathrm{J} 2$, could carry the pathogen from course to course, and even from region to region, where conditions favor establishment of the nematode.

\section{Acknowledgments}

Partial funding for this research was provided by our respective institutions. Additional funding was administered by the Northern California Golf Association with contributions from industry, individual golf courses, and professional associations. The photograph in Figure 2A was taken by G. Towers. The authors thank T. Cabasan, L. Duncan, C. Fleming, H. Helder, R. Ingham, Y. Oka, N. Sekora, J. Starr, and L. Waeyenberge for supplying root-knot nematode individuals and M. Hult for excellent technical assistance.

\section{Literature Cited}

1. Anonymous. 2009. Golf 20/20. In: World Golf Foundation. http:// www.golf2020.com/media/10053/economicimpact_2005golfeconomyreport 3.pdf.

2. Blok, V. C., and Powers, T. O. 2009. Biochemical and molecular identification. Pages 98-118 in: Root-Knot Nematodes. R. Perry, M. Moens, and J. Starr, eds. CABI Publishing, Wallingford, UK.

3. Blok, V. C., Wishart, J., Fargette, M., Berthier, K., and Phillips, M. S. 2002. Mitochondrial DNA differences distinguishing Meloidogyne mayaguensis from the major species of tropical root-knot nematodes. Nematology 4:773781.

4. Chen, P., Roberts, P. A., Metcalf, A. E., and Hyman, B. C. 2003. Nucleotide substitution patterning within the Meloidogyne rDNA D3 region and its evolutionary implications. J. Nematol. 35:404-410.

5. Cherry, T., Szalanski, A. L., Todd, T. C., and Powers, T. O. 1997. The internal transcribed spacer region of Belonolaimus (Nemata: Belonolaimidae). J. Nematol. 29:23-29.

6. Crow, W. T. 2005. How bad are nematode problems on Florida's golf courses? Florida Turf Dig. 22:10-12.

7. Crow, W. T. 2005. Plant-parasitic nematodes on golf course turf. Outlooks Pest Manage. (Pestic. Outlook) 16:10-15.

8. De Ley, I. T., De Ley, P., Vierstraete, A., Karssen, G., Moens, M., and Vanfleteren, J. 2002. Phylogenetic analyses of Meloidogyne small subunit rDNA. J. Nematol. 34:319-327.

9. De Ley, P., Tandigan De Ley, I., Morris, K., Abebe, E., Mundo-Ocampo, M., Yoder, M., Heras, J., Waumann, D., Rocha-Olivares, A., Burr, A. H. J., Baldwin, J. G., and Thomas, W. K. 2005. An integrated approach to fast and informative morphological vouchering of nematodes for applications in molecular barcoding. Philos. Trans. R. Soc. Ser. B 360:1945-1958.

10. Ferris, H., Carlson, H. L., Viglierchio, D. R., Westerdahl, B. B., Wu, F. W., Anderson, C. E., Juurma, A., and Kirby, D. W. 1993. Host status of selected crops to Meloidogyne chitwoodi. J. Nematol. 25:849-857.

11. Hanson, S. F., Solano, F., and Gil-Vega, K. K. 2009. An improved method for DNA sequence based identification of nematodes. (Abstr.) Phytopathology 99:S51.

12. Holterman, M., van der Wurff, A., van den Elsen, S., van Megen, H., Bongers, T., Holovachov, O., Bakker, J., and Helder, J. 2006. Phylum-wide analysis of SSU rDNA reveals deep phylogenetic relationships among nematodes and accelerated evolution toward crown clades. Mol. Biol. Evol. 23:1792-1800.

13. Howard, C. 2005. Nematodes? in Arizona ??? Cactus and Pine Golf Course Superintendents Association Newsletter May-June.

14. Huelsenbeck, J. P., and Ronquist, F. 2001. MRBAYES: Bayesian inference of phylogenetic trees. Bioinformatics 17:754-755. 
15. Hugall, A., Moritz, C., Stanton, J., and Wolstenholme, D. R. 1994. Low, but strongly structured mitochondrial-DNA diversity in root-knot nematodes (Meloidogyne). Genetics 136:903-912.

16. Jeanmougin, F., Thompson, J. D., Gibson, T. J., Plewniak, F., and Higgins, D. G. 1997. The CLUSTAL_X windows interface: flexible strategies for multiple sequence alignment aided by quality analysis tools. Nucleic Acids Res. 25:4876-4882.

17. Karssen, G., Bolk, R. J., Van Aelst, A. C., Van Den Beld, I., Kox, L. F. F., Korthals, G., Molendijk, L., Zijlstra, C., Van Hoof, R., and Cook, R. 2004. Description of Meloidogyne minor $\mathrm{n}$. sp. (Nematoda: Meloidogynidae), a root-knot nematode associated with yellow patch disease in golf courses. Nematology 6:59-72.

18. Landa, B. B., Rius, J. E. P., Vovlas, N., Carneiro, R. M. D. G., Maleita, C. M. N., Abrantes, I. M. O., and Castillo, P. 2008. Molecular characterization of Meloidogyne hispanica (Nematoda, Meloidogynidae) by phylogenetic analysis of genes within the rDNA in Meloidogyne spp. Plant Dis. 92:11041110.

19. Lyman, G. T., Throssell, C. S., Johnson, M. E., and Stacey, G. A. 2007. Golf course profile describes turfgrass, landscape, and environmental stewardship features. http://www.plantmanagementnetwork.org/sub/ats/research/ 2007/profile/.

20. MacGowan, J. B. 1984. Meloidogyne graminis, a root-knot nematode of grass. Fla. Dep. Agric. Consumer Serv. Div. Plant Ind. Circ. 107.

21. Milesi, C., Running, S. W., Elvidge, C. D., Dietz, J. B., Tuttle, B. T., and Nemani, R. R. 2005. Mapping and modeling the biogeochemical cycling of turf grasses in the United States. Environ. Manage. 36:426-438.

22. Obannon, J. H., Santo, G. S., and Nyczepir, A. P. 1982. Host range of the Columbia root-knot nematode. Plant Dis. 66:1045-1048.

23. Palomares Rius, J. E., Vovlas, N., Troccoli, A., Liebanas, G., Landa, B. B., and Castillo, P. 2007. A new root-knot nematode parasitizing Sea Rocket from Spanish Mediterranean coastal dunes: Meloidogyne dunensis n. sp. (Nematoda: Meloidogynidae). J. Nematol. 39:190-202.

24. Pokharel, R. R., Abawi, G. S., Zhang, N., Duxbury, J. M., and Smarti, C. D. 2007. Characterization of isolates of Meloidogyne from rice-wheat production fields in Nepal. J. Nematol. 39:221-230.

25. Powers, T. 2004. Nematode molecular diagnostics: from bands to barcodes. Annu. Rev. Phytopathol. 42:367-383.

26. Powers, T. O., and Harris, T. S. 1993. A polymerase chain reaction method for identification of five major Meloidogyne species. J. Nematol. 25:1-6.

27. Powers, T. O., Mullin, P. G., Harris, T. S., Sutton, L. A., and Higgins, R. S. 2005. Incorporating molecular identification of Meloidogyne spp. into a large-scale regional nematode survey. J. Nematol. 37:226-235.

28. Santo, G. S., O'Bannon, J. H., Finley, A. M., and Golden, A. M. 1980. Occurrence and host range of a new root-knot nematode (Meloidogyne chitwoodi) in the Pacific Northwest. Plant Dis. 64:951-952.

29. Sikora, E. J., Guertal, E. A., and Bowen, K. L. 2001. Plant-parasitic nematodes associated with hybrid bermudagrass and creeping bentgrass putting greens in Alabama. Nematropica 31:301-305.

30. Sites, J. W., and Marshall, J. C. 2004. Operational criteria for delimiting species. Annu. Rev. Ecol. Evol. Syst. 35:199-227.
31. Skantar, A. M., Handoo, Z. A., Carta, L. K, and Chitwood, D. J. 2007. Morphological and molecular identification of Globodera pallida associated with potato in Idaho. J. Nematol. 39:133-144.

32. Stanton, J., Hugall, A., and Moritz, C. 1997. Nucleotide polymorphisms and an improved PCR-based mtDNA diagnostic for parthenogenetic root-knot nematodes (Meloidogyne spp.). Fundam. Appl. Nematol. 20:261-268.

33. Stowell, L., and Gelernter W. 2008. Evaluation of nematode thresholds and turf damage. http://www.paceturf.org/index.php/journal/evaluation_of_ nematode thresholds and turf damage/.

34. Stowell, L. J., and McClure, M. A. 1993. A survey of plant-parasitic nematodes in Southern California golf course putting-green soils. (Abstr.) Phytopathology 83:1338.

35. Swofford, D. L. 2003. PAUP*. Phylogenetic Analysis Using Parsimony (*and other methods), Version 4. Sinauer Associates, Sunderland, MA.

36. Tenente, G. C. M. V., De Ley, P., De Ley, I. T., Karssen, G., and Vanfleteren, J. R. 2004. Sequence analysis of the D2/D3 region of the large subunit RDNA from different Meloidogyne isolates. Nematropica 34:1-12.

37. Thomas, W. K., Vida, J. T., Frisse, L. M., Mundo, M., and Baldwin, J. G. 1997. DNA sequences from formalin-fixed nematodes: integrating molecular and morphological approaches to taxonomy. J. Nematol. 29:250-254.

38. Tigano, M. S., Carneiro, R. M. D. G., Jeyaprakash, A., Dickson, D. W., and Adams, B. J. 2005. Phylogeny of Meloidogyne spp. based on 18S rDNA and the intergenic region of mitochondrial DNA sequences. Nematology 7:851862.

39. Todd, T. C., and Tisserat, N. A. 1990. Occurrence, spatial-distribution, and pathogenicity of some phytoparasitic nematodes on creeping bentgrass putting greens in Kansas. Plant Dis. 74:660-663.

40. Westerdahl, B. B., Harivandi, M.A., and Costello, L.R. 2005. Biology and management of nematodes on turfgrass in Northern California. USGA Greens Section Record September-October:7-10.

41. Xu, J. H., Liu, P. L., Meng, Q. P., and Long, H. 2004. Characterisation of Meloidogyne species from China using isozyme phenotypes and amplified mitochondrial DNA restriction fragment length polymorphism. Eur. J. Plant Pathol. 110:309-315.

42. Ye, W., Giblin-Davis, R. M., Davies, K. A., Purcell, M. F., Scheffer, S. J. Taylor, G. S., Center, T. D., Morris, K., and Thomas, W. K. 2007. Molecular phylogenetics and the evolution of host plant associations in the nematode genus Fergusobia (Tylenchida: Fergusobiinae). Mol. Phylogenet. Evol. 45:123-141.

43. Yoder, M., De Ley, I. T., King, I. W., Mundo-Ocampo, M., Mann, J., Blaxter, M., Poiras, L., and De Ley, P. 2006. DESS: a versatile solution for preserving morphology and extractable DNA of nematodes. Nematology 8:367-376.

44. Yu, Q., Potter, J. W., and Gilby, G. 1998. Plant-parasitic nematodes associated with turfgrass in golf courses in southern Ontario. Can. J. Plant Pathol. 20:304-307.

45. Zijlstra, C. 2000. Identification of Meloidogyne chitwoodi, M. fallax and M. hapla based on SCAR-PCR: A powerful way of enabling reliable identification of populations or individuals that share common traits. Eur. J. Plant Pathol. 106:283-290. 